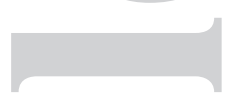

\title{
An efficient goal based reduced order model approach for targeted adaptive observations
}

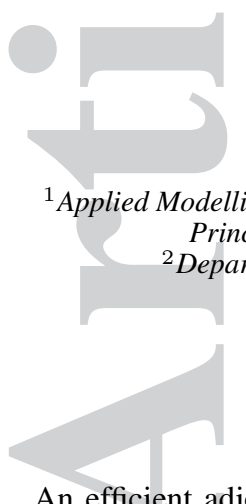

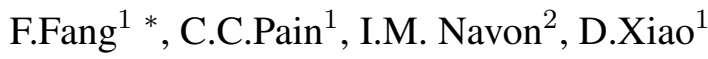

Prince Consort Road, London, SW7 2BP, URL: http: / / amcg.ese.imperial.ac.uk

Department of Scientific Computing, Florida State University, Tallahassee, FL, 32306-4120, USA

An efficient adjoint sensitivity technique for optimally collecting targeted observations is presented. The targeting technique incorporates dynamical information from the numerical model predictions to identify when, where, and what types of observations would provide the greatest improvement to specific model forecasts at a future time. A functional (goal) is defined to measure what is considered important in modelling problems. The adjoint sensitivity technique is used to identify the impact of observations on the predictive accuracy of the functional, then placing the sensors at the locations with high impacts. The adaptive (goal) observation technique developed here has the following features: (1) over existing targeted observation techniques, its novelty lies in that the interpolation error of numerical results is introduced to the functional (goal) which ensures the measurements are a distance apart; (2) the use of proper orthogonal decomposition (POD) and reduced order modeling (ROM) for both the forward and backward simulations, thus reducing the computational cost; and (3) the use of unstructured meshes.

The targeted adaptive observation technique, is developed here within an unstructured mesh finite element model (Fluidity). In this work, a POD ROM is used to form the reduced order forward model by projecting the original complex model from a high dimensional space onto a reduced order space. The reduced order adjoint model is then constructed directly from the reduced order forward model. This efficient adaptive observation technique has been validated with two test cases: a model of an ocean Gyre and a model of 2D urban street canyon flows. Copyright (c) 2016 John Wiley \& Sons, Ltd.

Received ...

KEY WORDS: Adaptive Observations, Finite Element, Proper orthogonal decomposition, Reduced order

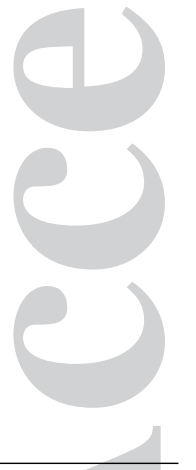
modelling.

${ }^{*}$ Correspondence to: Applied Modelling and Computation Group, Department of Earth Science and Engineering, Imperial College London, Prince Consort Road, London, SW7 2BP. E-mail: f.fang@imperial.ac.uk

This article has been accepted for publication and undergone full peer review but has not been through the copyediting, typesetting, pagination and proofreading process, which may lead to differences between this version and the Version of Record. Please cite this article as doi: $10.1002 / f l d .4265$ 


\section{INTRODUCTION}

Data assimilation is an efficient means of producing a best estimate model solution by fitting a numerical simulation to observational data over both space and time. The technique also facilitates the estimation of the error sources caused by uncertainties in the models. Data assimilation is achieved by minimising a cost function that reconciles the misfits between the data and dynamical modelling results, as well as the covariances specifying spatial and temporal correlations of errors [1-7].

There is a need to optimally place observations that will maximally improve the accuracy of numerical solutions at forecast times typical for the considered models. The resulting observation network could be adapted for a wide range of forecasting goals, and it could be adapted either by allocating existing observations differently or by adding observations from programmable platforms to the existing network [8,9,9-19,19-32]. Expensive field-deployed resources can thus be utilized more effectively by selecting an optimal observational network. Adaptive sensor location methods provide importance maps of solutions which will be convolved with the spatial and time density of observations in order to determine where best to place monitoring devices (i.e., components and location of the sensors). Reduction of errors can thus be achieved as well as assimilating fewer observational resources [10,24,33].

Given an energy norm or a goal (a functional or a single quantity of interest for example a component of the solution vector at an important location at a given instance in time), the targeting approach incorporates dynamical information from the numerical model predictions to identify when, where, and what types of observations would provide the greatest improvement to specific model forecasts at a future time. Such targeted observations are important as they will allow the most effective use of available monitoring resources. The approach initially involves the definition of an overall goal (functional), which is a measure of what is considered important in an environmental/physical problem. This approach will provide an optimal sensor location/network, uncertainty sensitivity analysis as well as quantification of the goal accuracy and will indicate where to increase or decrease numerical resolution (refine or de-refine the mesh).

The adjoint sensitivity analysis technique $[10,20,28,29,34]$ has proven to be an essential tool for developing adaptive observations strategies and will be used to help identify the areas where the errors in the uncertainties in models are rapidly growing and will mostly influence the forecast. This approach is highly complementary to recent advances in the development and deployment of portable and mobile wireless sensor networks [12]. The configuration of these systems can be dynamically adapted to transmit high resolution data in near real time from a selected set of locations to a centralised data store.

Many of the most powerful methods in these areas share a fundamental mathematical basis important for advanced threat detection: they rely on the adjoint model. All of the above techniques of predictive modelling depend on the availability of the adjoint model. However, the use of these adjoint-based techniques is very limited. The main reason is the extreme difficulty of developing adjoint models: the mathematical and computational expertise required to adjoint complex forward models is limited to handfuls of practitioners around the globe.

In this work, a reduced order method based on proper orthogonal decomposition (POD) has been used to implement the adjoint model (called adjoint of reduced order modelling in [35]), which provides a straightforward approach to overcoming one of the key difficulties in developing adjoint models. This provides a promising way to dramatically simplify the process of adjoint model development. In combination with the interpolation error in solutions, the targeted sensitivity technique provides a space-time importance map for optimising sensor locations.

The remainder of this article is organised as follows. The targeted observation technique is described in section 2. In section 3 the details of the POD reduced order forward and adjoint models are given. The adjoint sensitivity approach based on POD is discussed in section 4 . Section 5 provides numerical examples of studies on sensor locations. Conclusions are drawn in section 6 .

This article is protected by copyright. All rights reserved. 


\section{ADAPTIVE (TARGETED) OBSERVATIONS}

The aim of adaptive observations is to optimise the accuracy of a goal (functional) defined at the verification time over the verification domain.

\subsection{Goal or functional}

Suppose that the functional is represented as:

$$
\mathcal{J}\left(\mathbf{u}_{t_{v}}\right)=\int_{\Omega_{v}} f(\mathbf{u}) d \Omega
$$

where $\mathbf{u}_{v}$ denotes the solution of a variable $\mathbf{u}$ (velocity, pollutants for example) at the verification time $t_{v}$, and $\Omega_{v}$ is the verification domain. The function $f\left(\mathbf{u}_{v}\right)$ may be any derived quantity of solutions $\mathbf{u}_{v}$ (e.g, kinetic energy, vorticity, pollutant concentration) over $\Omega_{v}$ at the verification time $t_{v}$. In finite element methods, the variable $\mathbf{u}$ can be written:

$$
\mathbf{u}=\sum_{n=1}^{\mathcal{N}} N_{j} \mathbf{u}_{n}
$$

where $\mathcal{N}_{j}$ is the finite element basic function, $\phi_{n}$ is the solution of variable $\phi$ at node $j, \mathcal{N}$ is the number of nodes used over the computational domain $\Omega$.

\subsection{Impact of observations}

Assume that a model forecast during the computational period $\left[t_{0}, t_{v}\right]$ is given by

$$
\mathbf{u}_{t_{i}}=\mathcal{M}_{\left(t_{0}, t_{i}\right)}=\mathcal{M}_{t_{i}} \ldots \mathcal{M}_{t_{1}} \mathcal{M}_{t_{0}}\left(\mathbf{u}_{t_{0}}\right), \quad t_{i} \in\left[t_{0}, t_{v}\right]
$$

where $\mathbf{u}_{t_{i}}$ and $\mathbf{u}_{t_{0}}$ denote the solutions at time levels $t_{v}$ and $t_{0}$ respectively and $\mathcal{M}_{t_{i}}$ is the nonlinear model operator at time level $t_{i}$. A targeted time can be defined at variable time levels during $\left[t_{0}, t_{v}\right]$ (here defined at $t_{0}$ ), A target region is then defined at the targeted time, where the error of solutions is expected to cause a significant forecast error at the forecast verification time $t_{v}$. The adaptive (targeted) observation method is used to identify the area (targeted) where best to assimilate the data into the model, and thus improving the accuracy of solutions, by minimising the functional defined in (1):

$$
\min _{\mathbf{u}_{t_{0}}} \mathcal{J}\left(\mathbf{u}_{t_{0}}, \mathbf{u}_{t_{v}}\right)=\min _{\mathbf{u}_{t_{0}}} \int_{\Omega_{v}} f\left(\mathbf{u}_{t_{v}}\right) d \Omega
$$

\section{POD REDUCED ORDER ADJOINT MODEL}

\subsection{POD reduced forward model}

The model variables $\mathbf{U}$ are sampled at the checkpoints (defined time interval) during the simulation $\left[t_{1}, \ldots, t_{k}, \ldots, t_{K}\right]$ (where, $t_{K}=t_{v}$ is the verification time). They are also referred to as snapshots $\mathbf{U}=\left\{\mathbf{u}_{t_{k}}\right\}$ ( $K$ being the number of snapshots). POD constructs a set of orthogonal basis functions $\left\{\Phi_{m}\right\}, 1 \leq m \leq M$ such that it minimises [36]

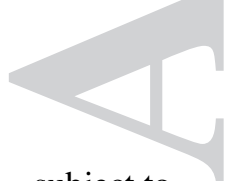

$$
\min \sum_{k=1}^{K}\left\|\mathbf{u}_{t_{k}}-\sum_{m=1}^{M} \Phi_{m} \Phi_{m}^{T} \mathbf{u}_{t_{k}}\right\|_{2}^{2},
$$

subject to

$$
\sum_{k=1}^{K}<\Phi_{m}, \Phi_{n}>_{2}=\delta_{m n}, \quad 1 \leq m, n \leq M \leq K,
$$

This article is protected by copyright. All rights reserved. 
where $<\cdot, \cdot>_{2}$ is the canonical inner product in $L^{2}$ norm and $M$ is the number of POD basis functions to be chosen.

In POD, the variable at time level $t_{i}$ in (3) can be expressed as an expansion of the POD basis functions $\Phi=\left(\Phi_{1}, \ldots, \Phi_{M}\right)$ :

$$
\mathbf{u}_{t_{i}}=\Phi \mathbf{u}_{t_{i}}^{r}=\sum_{m=1}^{M} \Phi_{m} u_{m, t_{i}}^{r}, \quad i=0, \ldots, K,
$$

where $\mathbf{u}^{r} \in R^{M}$ are a set of the time-dependent coefficients to be determined over the reduced space (the superscript $r$ indicates a variable or operator associated with the reduced order model). By using the Galerkin projection, the POD reduced system of equation (3) can be obtained:

$$
\mathbf{u}_{t_{v}}^{r}=\mathcal{M}_{t_{v}}^{r} \ldots \mathcal{M}_{t_{i}}^{r} \ldots \mathcal{M}_{t_{1}}^{r} \mathcal{M}_{t_{0}}^{r}\left(\mathbf{u}_{t_{0}}^{r}\right)=\mathcal{M}_{\left(t_{0}, t_{v}\right)}^{r}\left(\mathbf{u}_{t_{0}}^{r}\right), \quad t_{i} \in\left[t_{0}, t_{v}\right],
$$

where $\mathbf{u}_{t_{0}}^{r}=\Phi^{T} \mathbf{u}_{t_{0}}, \mathcal{M}_{\left(t_{0}, t_{v}\right)}^{r}=\mathcal{M}_{t_{v}}^{r} \ldots \mathcal{M}_{t_{i}}^{r} \ldots \mathcal{M}_{t_{1}}^{r} \mathcal{M}_{t_{0}}^{r}$ is the reduced order model operator, and

$$
\mathcal{M}_{t_{i}}^{r}=\Phi^{T} \mathcal{M}_{t_{i}}\left(\mathbf{u}_{t_{i}}^{r}\right) \Phi .
$$

It is time consuming to calculate $\mathcal{M}_{t_{i}}^{r}$ at each time level. To speed up the matrix equation assembly process, the matrix $\mathcal{M}_{t_{i}}^{r}$ is constructed here by a set of sub-matrices independent of time. For a quadratic nonlinear simulation, the full matrices $\mathcal{M}_{t_{i}}$ can be expressed as

$$
\mathcal{M}_{t_{i}}=\hat{\mathcal{M}}_{0}+\sum_{m=1}^{M} \hat{\mathcal{M}}_{m} u_{m, t_{i}}^{r} \in R^{\mathcal{N} \times \mathcal{N}},
$$

where $\hat{\mathcal{M}}_{0}$ and $\hat{\mathcal{M}}_{m}\left(\Phi_{m}\right)$ are time-independent operators. For calculation of $\hat{\mathcal{M}}_{0}$ and $\hat{\mathcal{M}}\left(\Phi_{m}\right)$, see [37]. For high order nonlinear problems, there is an error, $\sim(\delta u)^{3}$, in the POD solutions because of the high order non-linearity. However, in this paper there is only quadratic non-linearities and thus the current non-linear treatment is exact.

Projecting equation (10) onto the reduced space, yields [37]:

$$
\mathcal{M}_{t_{i}}^{r}=\hat{\mathcal{M}}_{0}^{r}+\sum_{m=1}^{M} \hat{\mathcal{M}}_{m}^{r} u_{m, t_{i}}^{r} \in R^{M \times M},
$$

where

$$
\hat{\mathcal{M}}_{0}^{r}=\Phi^{T} \hat{\mathcal{M}}_{0} \Phi, \quad \hat{\mathcal{M}}_{m}^{r}=\Phi^{T} \hat{\mathcal{M}}_{m} \Phi,
$$

where $\hat{\mathcal{M}}_{0}^{r}$ and $\hat{\mathcal{M}}_{m}^{r}(m=1, \ldots, M)$ are time-independent matrices and pre-computed.

\subsection{Discrete reduced order adjoint equations}

A small perturbation $\delta \mathbf{u}_{t_{0}}$ is given to the initial state of the full model, in turn, a small perturbation $\delta \mathbf{u}_{t_{0}}^{r}=\Phi^{T} \delta \mathbf{u}_{t_{0}}$ to the initial state of the reduced order model $\mathbf{u}_{t_{v}}^{r}=\mathcal{M}_{\left(t_{0}, t_{v}\right)}^{r}\left(\mathbf{u}_{t_{0}}^{r}+\delta \mathbf{u}_{t_{0}}^{r}\right)$. The discrete reduced order Tangent Linear Model (TLM) is obtained using the first order Taylor series:

$$
\delta \mathbf{u}_{t_{v}}^{r}=\frac{\partial \mathcal{M}_{\left(t_{0}, t_{v}\right)}^{r}\left(\mathbf{u}_{t_{0}}^{r}\right)}{\partial \mathbf{u}_{t_{0}}^{r}} \delta \mathbf{u}_{t_{0}}^{r}=\overline{\mathcal{M}}^{r} \delta \mathbf{u}_{t_{0}}^{r}=\overline{\mathcal{M}}_{t_{v}}^{r}, \ldots, \overline{\mathcal{M}}_{t_{i}}^{r}, \ldots, \overline{\mathcal{M}}_{t_{0}}^{r} \delta \mathbf{u}_{t_{0}}^{r},
$$

where

$$
\overline{\mathcal{M}}_{\left(t_{0}, t_{v}\right)}^{r}=\frac{\partial \mathcal{M}_{\left(t_{0}, t_{v}\right)}^{r}\left(\mathbf{u}_{t_{0}}^{r}\right)}{\partial \mathbf{u}_{t_{0}}^{r}}=\overline{\mathcal{M}}_{t_{v}}^{r}, \ldots, \overline{\mathcal{M}}_{t_{i}}^{r}, \ldots, \overline{\mathcal{M}}_{t_{0}}^{r} \in R^{M \times M}
$$

Taking into account (11), yields:

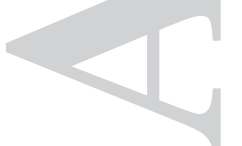

$$
\overline{\mathcal{M}}_{t_{i}}^{r}=\frac{\partial \mathcal{M}_{t_{i}}^{r}\left(\mathbf{u}_{t_{i}}^{r}\right)}{\partial \mathbf{u}_{t_{i}}^{r}}=\sum_{m=1}^{M} \hat{\mathcal{M}}_{m}^{r} \in R^{M \times M} .
$$

The adjoint of the tangent linear model (14) is the transpose of $\overline{\mathcal{M}}_{\left(t_{0}, t_{v}\right)}^{r}$, that is,

$$
\overline{\mathcal{M}}_{\left(t_{0}, t_{v}\right)}^{r, T}=\overline{\mathcal{M}}_{t_{0}}^{r, T}, \ldots, \overline{\mathcal{M}}_{t_{i}}^{r, T}, \ldots, \overline{\mathcal{M}}_{t_{v}}^{r, T} .
$$

This article is protected by copyright. All rights reserved. 


\section{OPTIMISATION OF SENSOR LOCATIONS BASED ON PROPER ORTHOGONAL}

\section{DECOMPOSITION}

\subsection{The functional (goal) and its gradient}

To measure the error in the numerical solution $\mathbf{u}_{t_{v}}$ at the verification time $t_{v}$, the functional (goal) in (1) is defined as follows:

$$
\mathcal{J}\left(\mathbf{u}_{t_{v}}, \mathbf{u}_{t_{0}}\right)=\frac{1}{2}\left(\mathbf{u}_{t_{v}, o}-\mathcal{H}\left(\mathbf{u}_{t_{v}}\right)\right)^{T} \mathbf{E}^{-1}\left(\mathbf{u}_{t_{v}, o}-\mathcal{H}\left(\mathbf{u}_{t_{v}}\right)\right),
$$

where $\mathbf{u}_{t_{v}, o}$ and $\mathbf{u}_{t_{v}}=\mathcal{M}_{t_{0}, t_{v}}\left(\mathbf{u}_{0}\right)$ represent the 'true' (or observational data) and numerical solutions (velocity, pressure, for example) at the certification time $t_{v}$ respectively; $\mathbf{E}$ is a diagonal error covariance matrix; $\mathcal{H}$ denotes the observational Gaussian operator.

Let us consider a first order derivation of the functional in equation (17) with respect to the initial coefficient $\mathbf{u}_{t_{0}}$. The gradient of the functional can be obtained:

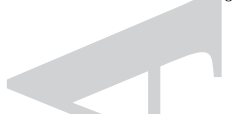

$$
\nabla_{\mathbf{u}_{t_{0}}} \mathcal{J}=\nabla_{\mathbf{u}_{t_{0}}} \mathcal{J}=\overline{\mathcal{M}}_{\left(t_{0}, t_{v}\right)}^{T} \mathbf{H}^{T} \mathbf{E}^{-1}\left(\mathbf{u}_{t_{v}, o}-\mathcal{H}\left(\mathbf{u}_{t_{v}}\right)\right),
$$

where

$$
\begin{gathered}
\overline{\mathcal{M}}_{\left(t_{0}, t_{v}\right)}=\frac{\partial \mathcal{M}_{\left(t_{0}, t_{v}\right)}\left(\mathbf{u}_{t_{0}}+\delta \mathbf{u}_{t_{0}}\right)}{\partial \mathbf{u}_{t_{0}}}=\overline{\mathcal{M}}_{t_{v}}, \ldots, \overline{\mathcal{M}}_{t_{i}}, \ldots, \overline{\mathcal{M}}_{t_{0}} \delta \mathbf{u}_{t_{0}} \\
\overline{\mathcal{M}}_{t_{i}}=\frac{\partial \mathcal{M}_{t_{i}}}{\partial \mathbf{u}_{t_{i}}}
\end{gathered}
$$

4.2. Adjoint sensitivity analysis using $P O D$

In POD, taking into account (7), equation (17) can be re-written:

$$
\mathcal{J}\left(\mathbf{u}_{t_{v}}^{r}, \mathbf{u}_{t_{0}}^{r}\right)=\frac{1}{2}\left(\mathbf{u}_{t_{v}, o}-\mathcal{H}\left(\Phi \mathbf{u}_{t_{v}}^{r}\right)\right)^{T} \mathbf{E}^{-1}\left(\mathbf{u}_{t_{v}, o}-\mathcal{H}\left(\Phi \mathbf{u}_{t_{v}}^{r}\right)\right),
$$

The first order derivation of the functional in equation (21) with respect to the initial coefficient $\mathbf{u}_{0}^{r}$ over the reduced order space can be obtained:

$$
\nabla_{\mathbf{u}_{t_{0}}^{r}} \mathcal{J}=\frac{\partial \mathcal{J}}{\partial \mathbf{u}_{t_{0}}^{r}}=\overline{\mathcal{M}}_{\left(t_{0}, t_{v}\right)}^{r, T} \Phi \mathbf{H}^{T} \mathbf{E}^{-1}\left(\mathbf{u}_{t_{v}, o}-\mathcal{H}\left(\Phi \mathbf{u}_{t_{v}}^{r}\right)\right)
$$

where $\overline{\mathcal{M}}_{\left(t_{0}, t_{v}\right)}^{T}$ is the adjoint of the tangent linear model (see equation (16)). The impact of each solution variable $u_{n, t_{i}}$ on the predictive accuracy of the functional can be obtained:

$$
\delta \mathcal{J}_{u_{n}, t_{0}}=\left(\nabla_{u_{n, t_{0}}} \mathcal{J}\right) \delta u_{n, t_{0}},
$$

where $n \in\{1, \ldots, \mathcal{N}\}(\mathcal{N}$ is the number of nodes over the domain). This provides a space-time importantance map of solutions over the space and time which will be convolved with the spatial and time density of observations in order to determine where best to place monitoring devices.

\subsection{Interpolation error in solutions}

An estimate of errors in $\mathbf{u}_{t_{0}}=\left(u_{1, t_{0}}, \ldots, u_{\mathcal{N}, t_{0}}\right)$ is used to determine the contribution of solutions to the error in $\mathcal{J}$. Using the interpolation theory, the error in $\mathbf{u}_{t_{0}}$ can be estimated:

$$
\delta \mathbf{u}_{t_{0}}(\mathbf{x})=\left|\mathbf{u}_{t_{0}, o}-\mathbf{u}_{t_{0}}\right| \approx \min _{o=1}^{D}\left(\mathbf{x}-\mathbf{x}_{o}\right)^{T} \frac{1}{2}\left(|Q(\mathbf{x})|+\left|Q\left(\mathbf{x}_{o}\right)\right|\right)\left(\mathbf{x}-\mathbf{x}_{o}\right),
$$

where $\mathbf{u}_{t_{0}, o}$ is the observational data; $\mathbf{x}_{o}$ is the observational point $o ; D$ is the number of observational data available over the domain; $Q$ is the Hessian matrix of variable fields $\mathbf{u}_{t_{0}}$. For 
one dimensional problems, $Q=\frac{\partial^{2} \mathbf{u}_{0}}{\partial x^{2}}$, while for three dimensional problems:

$$
Q=\left(\begin{array}{ccc}
\frac{\partial^{2} \mathbf{u}_{t_{0}}}{\partial x^{2}} & \frac{\partial^{2} \mathbf{u}_{t_{0}}}{\partial x \partial y} & \frac{\partial^{2} \mathbf{u}_{t_{0}}}{\partial x \partial z} \\
\frac{\partial^{2} \mathbf{u}_{t_{0}}}{\partial y \partial x} & \frac{\partial^{2} \mathbf{u}_{t_{0}}}{\partial y^{2}} & \frac{\partial^{2} \mathbf{u}_{t_{0}}}{\partial x \partial z} \\
\frac{\partial^{2} \mathbf{u}_{t_{0}}}{\partial z \partial x} & \frac{\partial^{2} \mathbf{u}_{t_{0}}}{\partial z \partial y} & \frac{\partial^{2} \mathbf{u}_{t_{0}}}{\partial z^{2}}
\end{array}\right)
$$

The absolute value of the symmetric Hessian matrix is defined as [38]:

$$
|Q|=\mathbf{V}|\vec{\Lambda}| \mathbf{V}^{\mathrm{T}},
$$

where, the matrices $\mathbf{V}$ and $\vec{\Lambda}$ contain the eigenvectors $\vec{e}_{k}$ and eigenvalues $\lambda_{k}$ of the Hessian matrix $\mathcal{H}$ respectively. The introduction of interpolations errors into the functional (a heuristic technique) ensures the sensors are not co-located. If there are no observations for a particular field within a multi-field solution variable then one may simple use the difference between the maximum and minimum values in the solution as the error in order to determine if it is worth observing a particular field e.g. pollutants.

\subsection{Typical algorithm for adaptive observations}

(a) Run the forward model that predicts the functional $\mathcal{J}$ at the verification time, by assimilating the data when/where available at sensors;

(b) Determine $\nabla_{\mathbf{u}_{t_{0}}} \mathcal{J}$ in equation (18) which provides the priority of each solution variable $u_{n, t_{0}}$;

(c) Obtain an approximation to the error in the solution $\delta u_{i, t_{0}}, e . g$. using the interpolation theory;

(d) Form the convolution $\delta \mathcal{J}_{u_{n}, t_{0}}=\left(\nabla_{u_{n, t_{0}}} \mathcal{J}\right) \delta u_{n, t_{0}}$;

(e) Choose the maximum value of $\delta \mathcal{J}_{n}$, and place a sensor at this position;

(f) Repeat (c) and (d) above until the required number of sensors is attained;

(g) Repeat the whole process until the required accuracy of $\mathcal{J}$ is attained.

\section{CASE STUDIES}

\subsection{An unstructured mesh finite element fluid model (Fluidity)}

The adjoint sensitivity approach in combination with reduced order modelling has been implemented within a 3D unstructured mesh finite element model (developed by the applied modelling and computation group at Imperial College London), which is capable of:

- solving the Navier-Stokes equations;

- use of a non-hydrostatic solver to model dense water formation and flow over steep topography; and

- anisotropic unstructured meshes in the vertical as well as the horizontal to capture the details of local flow in all three directions.

In this work, the adjoint model is implemented using POD-ROM, where the interpolation error is taken into account in the goal-based sensitivity formulation. A comparative study on sensor locations has been carried out using two test cases: Munk Gyre in ocean modelling and tracer dispersion in an urban street canyon.

This article is protected by copyright. All rights reserved. 


\subsection{Case 1: Munk gyre}

The adaptive sensor location technique is first demonstrated in the gyre problem (one frequently used problem in ocean modelling), a circulation flow driven by a wind force on the free surface. The computational domain is $1000 \mathrm{~km}$ by $1000 \mathrm{~km}$ with a depth of $H=500 \mathrm{~m}$. The wind forcing on the free surface is given by

$$
\tau_{y}=\tau_{0} \cos (\pi y / L), \quad \tau_{x}=0.0,
$$

where $\tau_{x}$ and $\tau_{y}$ are the wind stresses on the free surface along the $x$ and $y$ directions respectively, and $L$ is the size of the computational domain, here $L=1000 \mathrm{~km}$. A maximum zonal wind stress of $\tau_{0}=0.1 \mathrm{Nm}^{-1}$ is applied in the latitudinal $(y)$ direction. The Coriolis terms are taken into account using the beta-plane approximation $(f=\beta y)$ where $\beta=1.8 \times 10^{-11}$ and the reference density is $\rho_{0}=1000 \mathrm{kgm}^{-1}$.

Non-hydrostatic Boussinesq equations are solved in this case. The spin-up period is 8 days. The solution at $t=8$ day is taken as the initial state for the forward reduced order model. The computational period is $[8,66]$ days. The time step is $\Delta t=8 \mathrm{~h}$. The element length of $20 \mathrm{~m}$ is used. 50 snapshots of the solution from the full modelling are recorded during the simulation period $[8,66]$ day and from these snapshots 12 POD basis functions are generated for the velocity components $u, v, w$ and the pressure $p$ and capture more than $99 \%$ of energy in original kinetic energy. Both the reduced forward and adjoint models are formed based on these 12 POD basis functions.

The twin experiment scheme is employed in the data assimilation framework. Pseudoobservational data is generated from the forward simulation driven by a 'true' initial condition at $t=8$ day. The guessed value of initial conditions is taken from the forward solution at the $20^{t h}$ day. The goal functional at the verification time is defined in equation (17) over the verification domain. In this case, $\mathbf{u}$ in equation (17) represents the velocity field. The verification time level is $t_{v}=60$ day while the verification region is defined $\Omega_{v}=[150,350] \mathrm{km} \times[550,800] \mathrm{km}$ (the small rectangle shown in Figure 1) where a large error in solutions occurs.

The sensitivity of the functional with respect to the velocity solution at the verification time $t=8$ day was obtained by running the adjoint model backwards from day 66 to day 8 . Taking into account the interpolation error of solutions, the impact of the initial state on the accuracy of the targeted functional is plotted in Figure 2(a) and the corresponding optimal locations of sensors in Figure 2(b).

Figure 1 shows the velocity field and its error on day 66 before and after assimilating the data into the model. It illustrates the contribution of optimal data to improving the accuracy of numerical solutions. By assimilating the data collected at the first 100 optimal locations into the model $t=8 d a y$, the error in the velocity field on day 66 is significantly reduced over the verification region.

Figure 3 demonstrates the impact of data taken at a number of sensor locations on the solutions at $t=66$ day. The circle and star points represent the randomly collected and optimal data respectively. It can be seen the maximum error is reduced by $25 \%$ if 100 optimal sensors are used in data assimilation and further to half with use of 150 optimal sensors. The accuracy of numerical results is improved with the increased number of targeted data. However, it is not always this case if the data is not chosen optimally. We can see the accuracy may be not improved after assimilating the data collected randomly in the domain.

\subsection{Case 2: 2D urban street canyon flows}

The targeted adjoint sensitivity approach is further applied to the simulation of 2D street canyon flows. This test case is dimensionless. The geometry of the problem is rectangular in shape, with a length of 2 and a height of 1 , and contains two neighboring buildings that form a canyon between them (see Figure 4). A uniform velocity of 1 is prescribed on the inflow boundary located on the left side of the domain. No-slip boundary conditions are applied on the domain's bottom edge and all building surfaces. A free-slip condition is applied on the domain's top boundary. The kinematic viscosity is set to $1 \times 10^{-4}$ and the Reynolds number equates to $1 \times 10^{4}$.

This article is protected by copyright. All rights reserved. 
The spin-up period was set to 0.76 allowing the eddies to form. Following this the full simulation was run to $t=1.56$ using a time step of 0.008 . Over this time period $[0.76,1.56]$, forty snapshots were taken from the pre-computed solution at every tenth time step. From these snapshots 40 POD basis functions were constructed for each variable, and these bases were capable of capturing $99 \%$ of their energy whilst reducing the size of the problem by a factor of 200. Again, the forward and adjoint reduced order models are formed from these POD bases.

The data assimilation system is setup as follows: (1) the observational data for data assimilation is obtained by the twin experiment, that is, running the forward model forced by the 'true' initial condition at time instance 0.76 ; (2) the guessed initial condition is taken from the forward model solution at time instance 0.8 ; (3) the guessed initial condition is optimised by assimilating the data which is collected at the optimal sensor locations. The sensor locations are chosen using the adaptive observation approach i.e. minimising the (goal) functional in equation (17) which is defined as the error in velocity field at the verification time $(t=1.56)$ over the verification domain, $\Omega_{v}=[0.6,0.9] m \times[0.15,0.4]$ (see the small rectangle area in Figure 5).

The adjoint sensitivity approach using POD (see equations (22) and (18)) is used to identify the impact of solutions at time instance 0.75 on the predictive results over the verification region at $t=1.56$. The corresponding results are shown in Figure 6(a). The optimal sensor locations can thus be decided from large sensitivity values. By assimilating the data collected at the optimal sensor location, the error of solutions at the verification time $t=1.54$ is largely decreased over the verification region, which is shown in Figure 5 (b). With the use of POD ROM during the forward and backward integration, the problem size is reduced by factors of 200 in comparison with the high fidelity model.

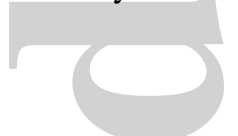

\section{CONCLUSIONS}

In this work, an efficient goal based reduced order model approach for targeted adaptive observations is developed within an unstructured mesh finite element model (Fluidity). For computational efficiency, POD ROM has been used in the forward model while the adjoint of the POD ROM has been used to form the sensitivities. The targeted sensitivity technique is used to identify high impact of observation on a defined functional (goal). A key novelty in the present study is the way we introduce structure or interpolation error into the sensor placement which ensures that the sensors are placed an optimal distance from each other.

The following conclusions can be drawn from the results represented here. The use of the adjoint targeted sensitivity technique in the data assimilation system can provide an important map for optimisation of sensor locations. The use of POD ROM can significantly reduce both the computer memory requirement and CPU time (by several orders of magnitude). It is also demonstrated that by assimilating the targeted data, the inputs (for example, initial conditions) can be optimised, thus improving the predictive accuracy of solutions at the verification time.

\section{ACKNOWLEDGEMENTS}

This work was carried out under funding from the UK's Natural Environment Research Council (project NE/J015938/1), the Engineering and Physical Sciences Research Council (EP/I00405X/1), Managing air for greener inner cities (MAGIC) (EP/N010221/1) and with support from the Imperial College High Performance Computing Service and the Grantham Institute for Climate Change. Dr. Xiao acknowledges the support of NSFC grant 11502241 and China postdoctoral science foundation grant (2014M562087). Prof. Pain acknowledges the support from MEMPHIS project. Prof. I. M. Navon acknowledges the support of NSF/CMG grant ATM-0931198.

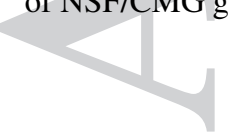

\section{REFERENCES}

[1] A. M. Moore, N. S. Cooper, and D. L. T. Anderson, Initialization and data assimilation in models of the Indian Ocean, J. Phys. Oceanogr. 17 (1987), 1965-1977.

This article is protected by copyright. All rights reserved. 
[2] A. F. Bennett, Chua B. S., D. E. Harrison, and M. J. McPhaden, Generalized inversion of Tropical AtmosphereOcean (TAO) data and a coupled model of the tropical Pacific. Part II: The 1995-96 La Nina and 1997-98 El Nino, J. Climate 13 (2000), no. 15, 2770-2785.

[3] W. C. Thacker and R. B. Long, Fitting dynamics to data, J. Geophys. Res. 93 (1988), no. C2, 1227-1240.

[4] E. Tziperman, W. C. Thacker, R. B. Long, S. M. Hwang, and S. R. Rintoul, Oceanic data analysis using a general circulation model. Part II: A North Atlantic model, J. Phys. Oceanog. 22 (1992), no. 12, 1458-1485.

[5] F Fang, CC Pain, IM Navon, MD Piggott, GJ Gorman, PE Farrell, PA Allison, and AJH Goddard, A POD reducedorder $4 D$-Var adaptive mesh ocean modelling approach, International Journal for Numerical Methods in Fluids 60 (2009), no. 7, 709-732.

[6] Dacian N Daescu and Ricardo Todling, Adjoint estimation of the variation in model functional output due to the assimilation of data, Monthly Weather Review 137 (2009), no. 5, 1705-1716.

[7] S-H Lee, S-W Kim, WM Angevine, L Bianco, SA McKeen, CJ Senff, M Trainer, SC Tucker, and RJ Zamora, Evaluation of urban surface parameterizations in the wrf model using measurements during the texas air quality study 2006 field campaign, Atmospheric Chemistry and Physics 11 (2011), no. 5, 2127-2143.

[8] MJ Hossen, IM Navon, and Fangxin Fang, A penalized 4-d var data assimilation method for reducing forecast error related to adaptive observations, International Journal for numerical methods in fluids 70 (2012), no. 10, $1207-1220$.

[9] MJ Hossen, IM Navon, and Dacian N Daescu, Effect of random perturbations on adaptive observation techniques, International Journal for Numerical Methods in Fluids 69 (2012), no. 1, 110-123.

[10] Dacian N Daescu and Ionel Michael Navon, Adaptive observations in the context of $4 d$-var data assimilation, Meteorology and Atmospheric Physics 85 (2004), no. 4, 205-226.

[11] Serge Gratton, M Monserrat Rincon-Camacho, and Philippe L Toint, Adaptive observations and multilevel optimization in data assimilation, Technical report, University of Namur (2013).

[12] Jeffrey C Neal, Peter M Atkinson, and Craig W Hutton, Adaptive space-time sampling with wireless sensor nodes for flood forecasting, Journal of hydrology 414 (2012), 136-147.

[13] Dariusz Uciński, Sensor network scheduling for identification of spatially distributed processes, International Journal of Applied Mathematics and Computer Science 22 (2012), no. 1, 25-40.

[14] Maciej Patan, Optimal sensor networks scheduling in identification of distributed parameter systems, Vol. 425, Springer Science \& Business Media, 2012.

[15] Sonja Wogrin, Model reduction for dynamic sensor steering: a bayesian approach to inverse problems, Ph.D. Thesis, 2008.

[16] Paritosh Mokhasi and Dietmar Rempfer, Optimized sensor placement for urban flow measurement, Physics of Fluids (1994-present) 16 (2004), no. 5, 1758-1764.

[17] Dacian N Daescu, On the sensitivity equations of four-dimensional variational (4d-var) data assimilation, Monthly Weather Review 136 (2008), no. 8, 3050-3065.

[18] Dacian N Daescu and Gregory R Carmichael, An adjoint sensitivity method for the adaptive location of the observations in air quality modeling, Journal of the Atmospheric Sciences 60 (2003), no. 2, 434-450.

[19] Junjie Liu and Eugenia Kalnay, Estimating observation impact without adjoint model in an ensemble kalman filter, Quarterly Journal of the Royal Meteorological Society 134 (2008), no. 634, 1327-1335.

[20] Humberto C Godinez and Dacian N Daescu, Observation targeting with a second-order adjoint method for increased predictability, Computational Geosciences 15 (2011), no. 3, 477-488.

[21] I Yu Gejadze and V Shutyaev, On computation of the design function gradient for the sensor-location problem in variational data assimilation, SIAM Journal on Scientific Computing 34 (2012), no. 2, B127-B147.

[22] Adrian Sandu, Emil Constantinescu, Gregory Carmichael, Tianfeng Chai, Dacian Daescu, and John Seinfeld, Ensemble methods for dynamic data assimilation of chemical observations in atmospheric models, Journal of Algorithms \& Computational Technology 5 (2011), no. 4, 667-692.

[23] Alexandru Cioaca, Adrian Sandu, and Eric de Sturler, Efficient methods for computing observation impact in 4d-var data assimilation, Computational Geosciences 17 (2013), no. 6, 975-990.

[24] Alexandru Cioaca and Adrian Sandu, An optimization framework to improve 4d-var data assimilation system performance, Journal of Computational Physics 275 (2014), 377-389.

[25] _ Low-rank approximations for computing observation impact in 4d-var data assimilation, Computers \& Mathematics with Applications 67 (2014), no. 12, 2112-2126.

This article is protected by copyright. All rights reserved. 
[26] Dariusz Uciński and Maciej Patan, Sensor network design for the estimation of spatially distributed processes, International Journal of Applied Mathematics and Computer Science 20 (2010), no. 3, 459-481.

[27] Raheam A Mansor Al-Saphory, Strategic sensors and regional exponential observability, ISRN Applied Mathematics 2011 (2011).

[28] Sharanya J Majumdar, SD Aberson, CH Bishop, Carla Cardinali, Jim Caughey, Alexis Doerenbecher, P Gauthier, R Gelaro, TM Hamill, RH Langland, et al., Targeted observations for improving numerical weather prediction: an overview, World Weather Research Programme/THORPEX Publication 15 (2011).

[29] Amy L Stuart, Altug Aksoy, Fuqing Zhang, and John W Nielsen-Gammon, Ensemble-based data assimilation and targeted observation of a chemical tracer in a sea breeze model, Atmospheric Environment 41 (2007), no. 14, 3082-3094.

[30] K. Cohen, S. Siegel, and T. Mclaughlin, Sensor placement based on poper orthogonal decomposition modeling of a cylinder wake, AIAA 2003-4259 (2003), 1-11.

[31] N.O. Song and D. Teneketzis, Discrete search with multiple sensors, Math Meth Oper Res 60 (2004), 1-13.

[32] AK Alekseev and IM Navon, Criteria of optimality for sensors' location based on adjoint transformation of observation data interpolation error, International journal for numerical methods in fluids 62 (2010), no. 1, 74 89.

[33] Z. Che, F. Fang, J. Percival, C.C.Pain, O. Matar, and I.M. Navon, An ensemble method for sensor optimisation applied to falling liquid films, International Journal of Multiphase Flow 67 (2014), 153-161.

[34] A. Sandu, D. N. Daescu, and G. R. Carmichael, Direct and adjoint sensitivity analysis of chemical kinetic systems with KPP: Part II - numerical validation and applications, Atmos. Environ. 37 (2003), no. 36, 5097-5114.

[35] Răzvan Ştefănescu, Adrian Sandu, and Ionel Michael Navon, POD/DEIM reduced-order strategies for efficient four dimensional variational data assimilation, Journal of Computational Physics 295 (2015), 569-595.

[36] Stefan Volkwein, Model reduction using proper orthogonal decomposition, Lecture Notes, Institute of Mathematics and Scientific Computing, University of Graz. see http://www. uni-graz. at/imawww/volkwein/POD. pdf (2011).

[37] Juan Du, Fangxin Fang, Christopher C Pain, I.M. Navon, Jiang Zhu, and David A Ham, POD reduced-order unstructured mesh modeling applied to $2 d$ and $3 d$ fluid flow, Computers and Mathematics with Applications $\mathbf{6 5}$ (2013), no. 3, 362-379.

[38] C. C. Pain, A. P. Umpleby, C. R. E. de Oliveira, and A. J. H. Goddard, Tetrahedral mesh optimisation and adaptivity for steady-state and transient finite element calculations, Comput. Methods Appl. Mech. Engrg. 190 (2001), 37713796.
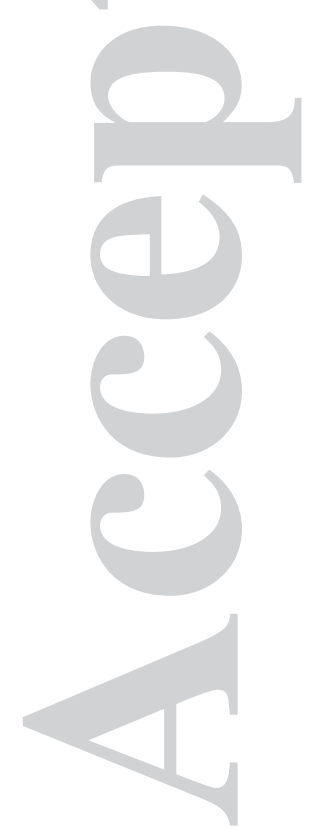

This article is protected by copyright. All rights reserved. 

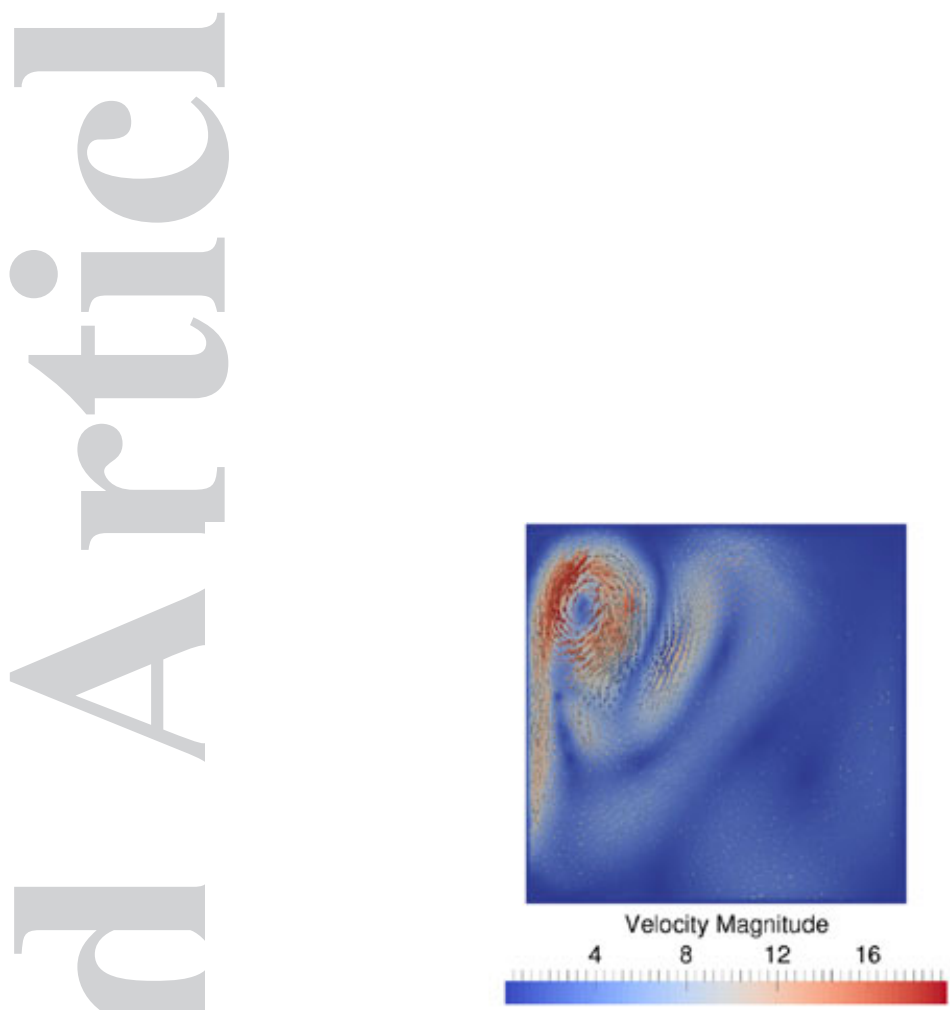

(a) Velocity field

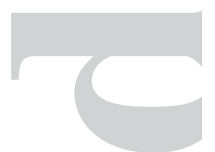

(a) Velocity field
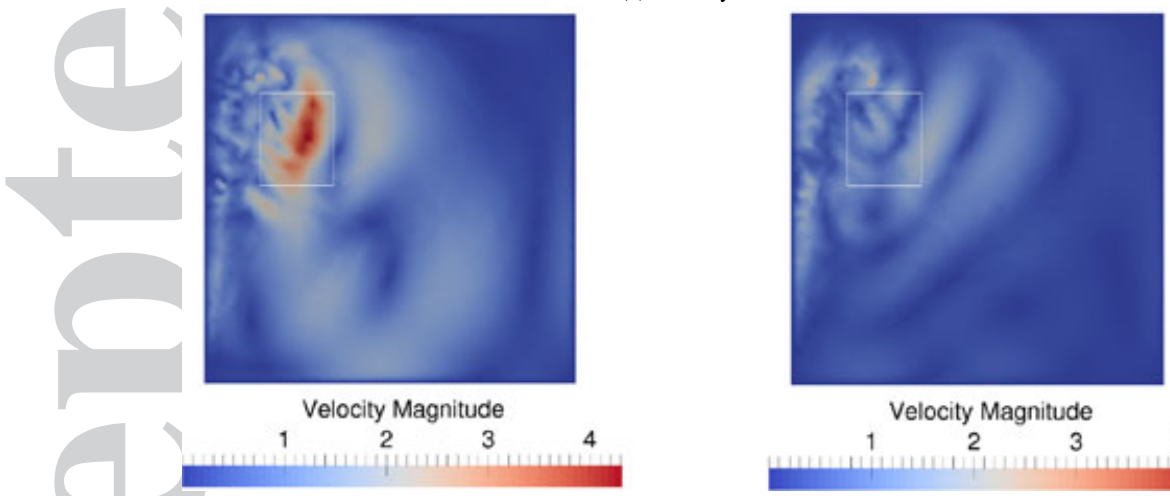

(b) Error in velocity solutions without data assimilation (c) Error in velocity solutions after assimilating 150 targeted data

Figure 1. Case 1 - Gyre: Velocity field and error in velocity solutions at the verification time level $(t=$ 66 day) with and without data assimilation.

This article is protected by copyright. All rights reserved. 


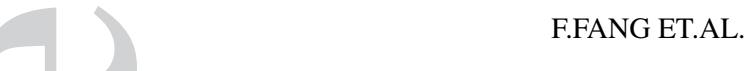

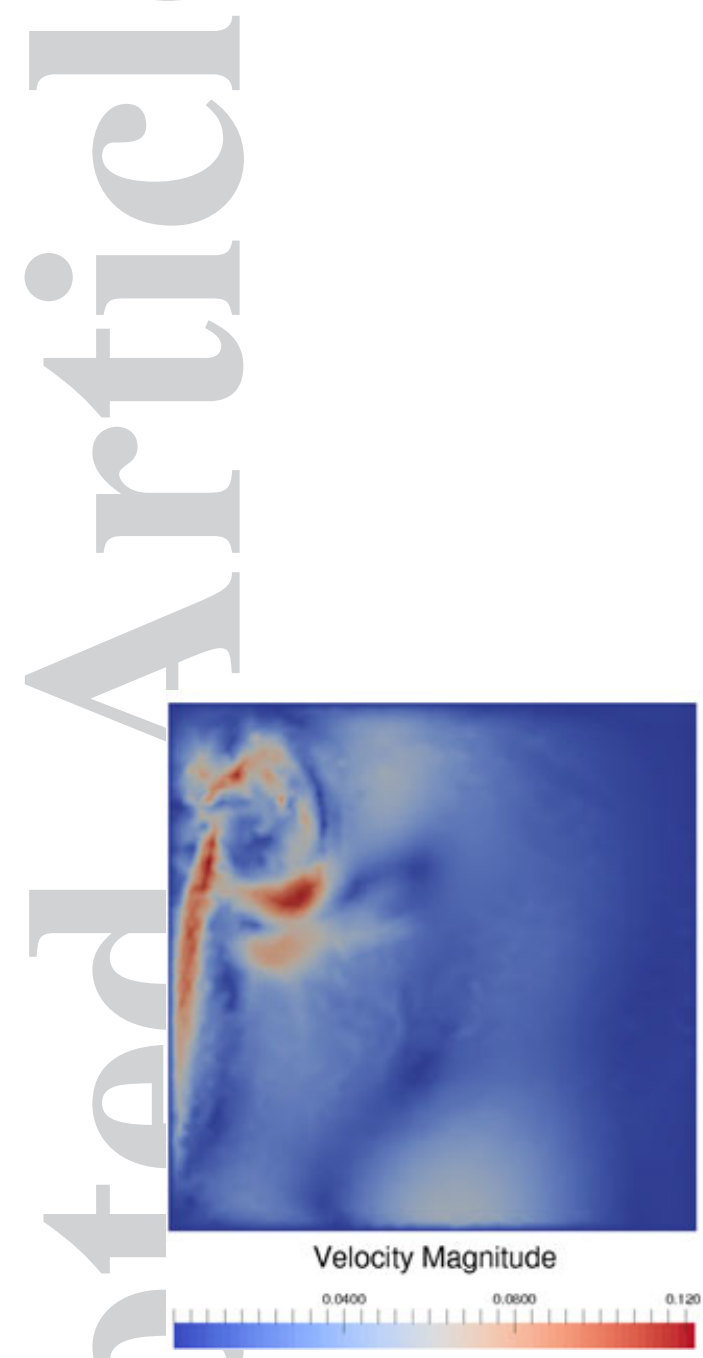

(a)Adjoint sensitivity solutions

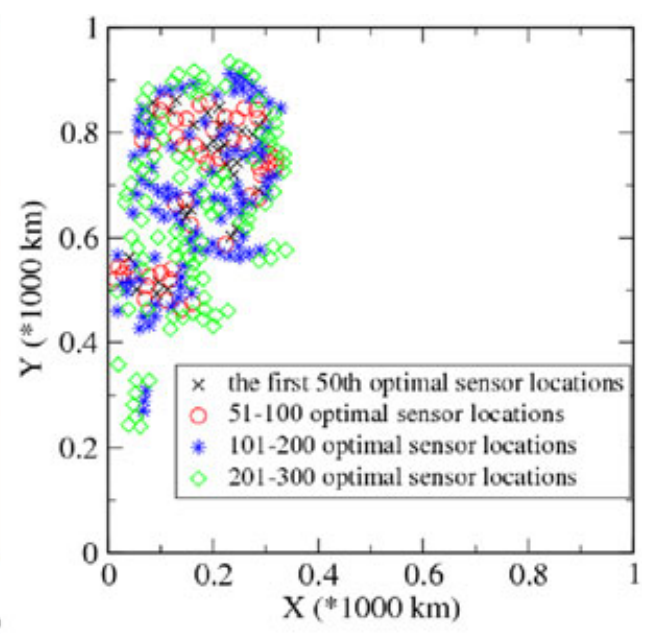

(b) Optimal sensor locations

Figure 2. Case 1 - Gyre: Optimisation of sensor locations. Left panel: adjoint sensitivity of the functional defined in (17) with respect to the solutions on day 8; right panel: optimal sensor locations.

This article is protected by copyright. All rights reserved. 


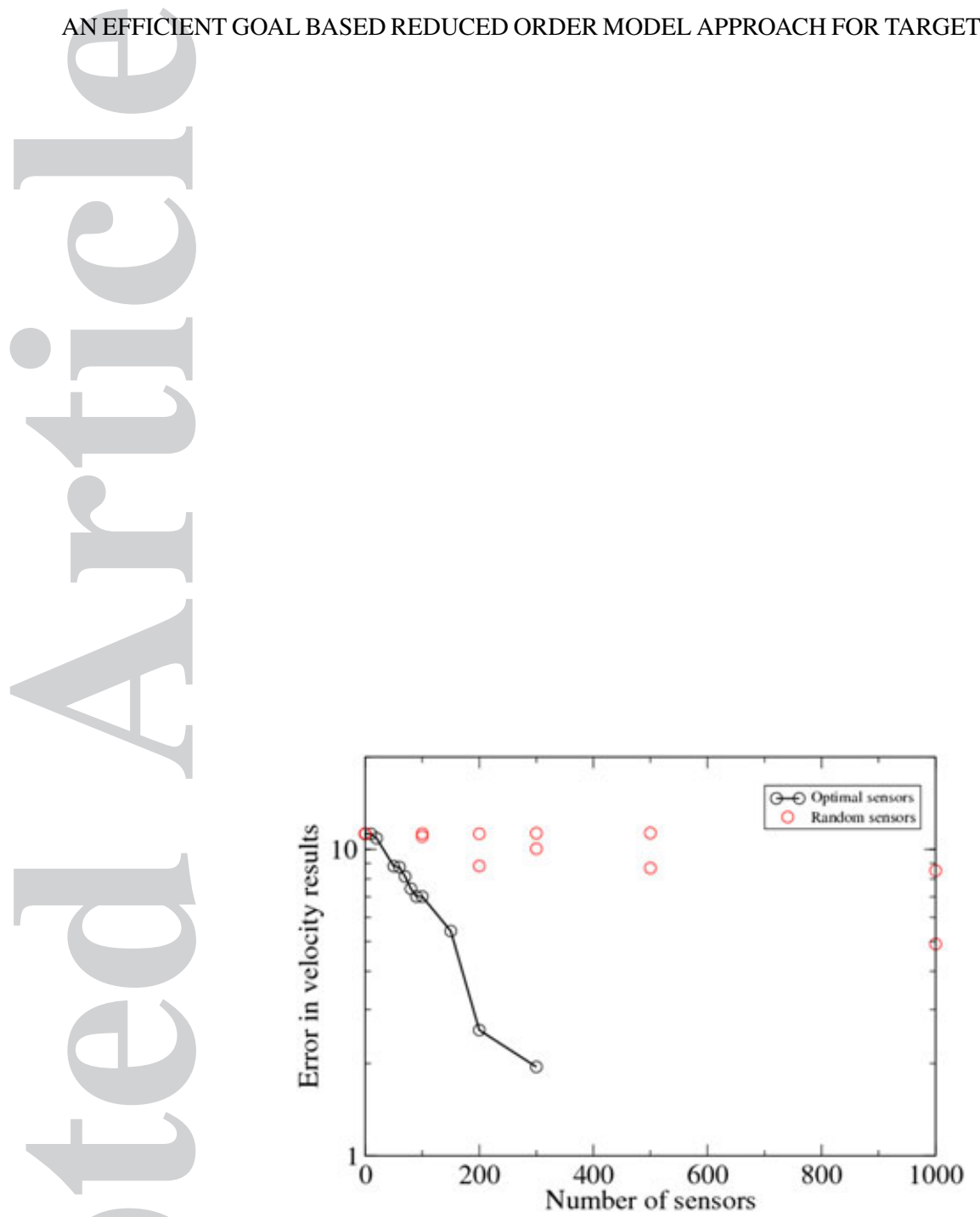

Figure 3. Case 1 - Gyre: The maximum error in velocity solutions at the verification time and region after assimilating the data into the model with and without optimising the sensor locations. The circle and star points represent the randomly collected and optimal data respectively.

This article is protected by copyright. All rights reserved. 


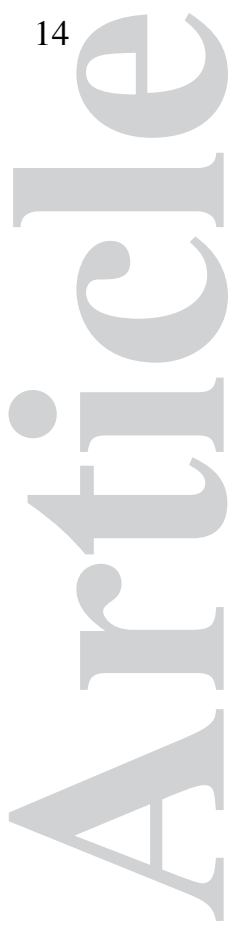

F.FANG ET.AL.

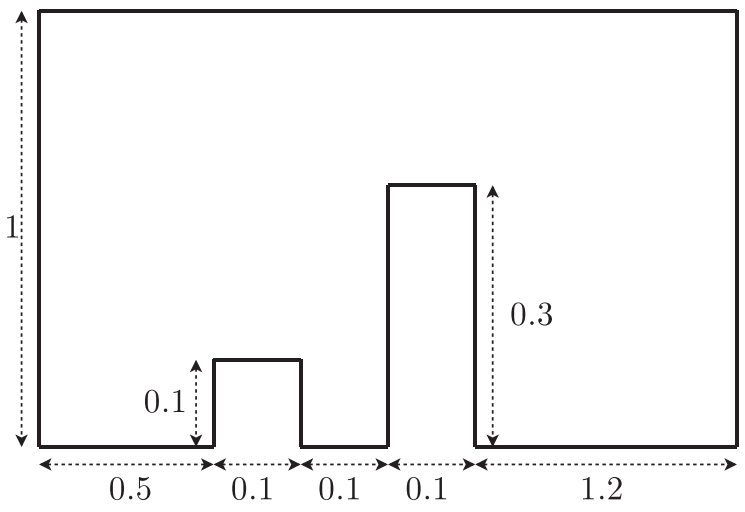

Figure 4. Case 2: Computational domain.

This article is protected by copyright. All rights reserved. 


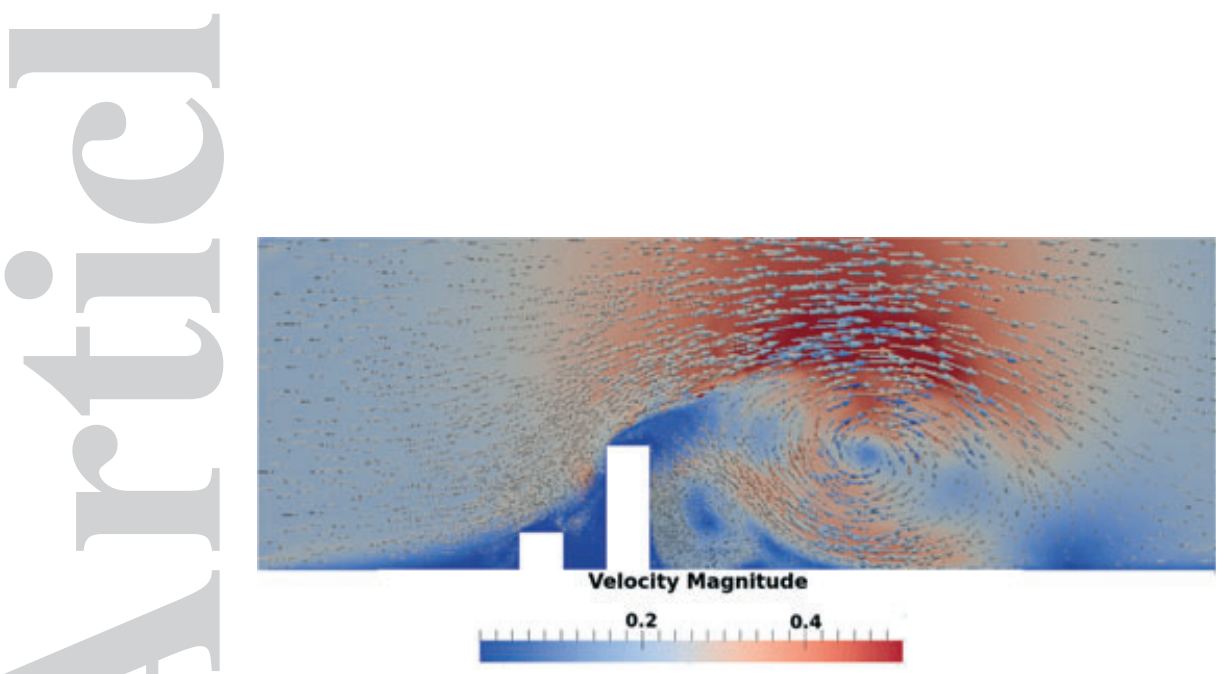

(a) Velocity field at $t=1.56$

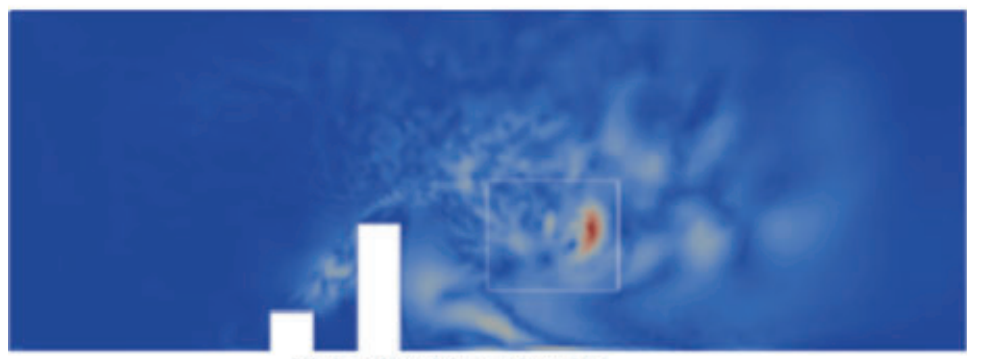

Velocity Magnitude

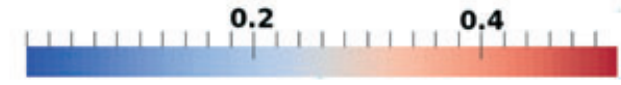

(b) Error in velocity solutions at $t=1.56$ without using data assimilation techniques

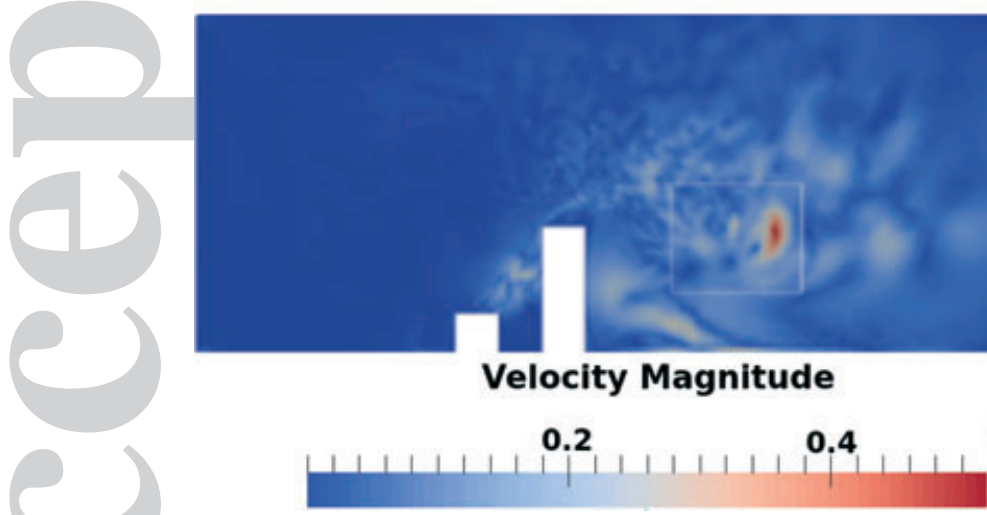

(c) Error in velocity solutions at $t=1.56$ with using data assimilation techniques

Figure 5. Case 2: Velocity field and error in velocity solutions at the verification time level with and without data assimilation.

This article is protected by copyright. All rights reserved. 


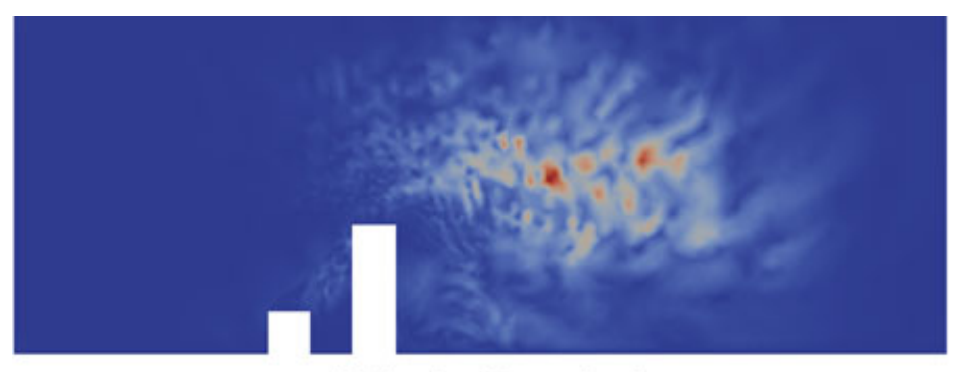

Velocity Magnitude

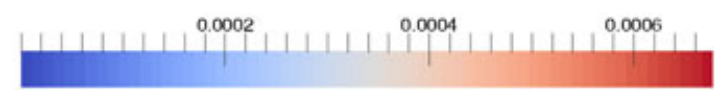

(a) Sensitivity solution
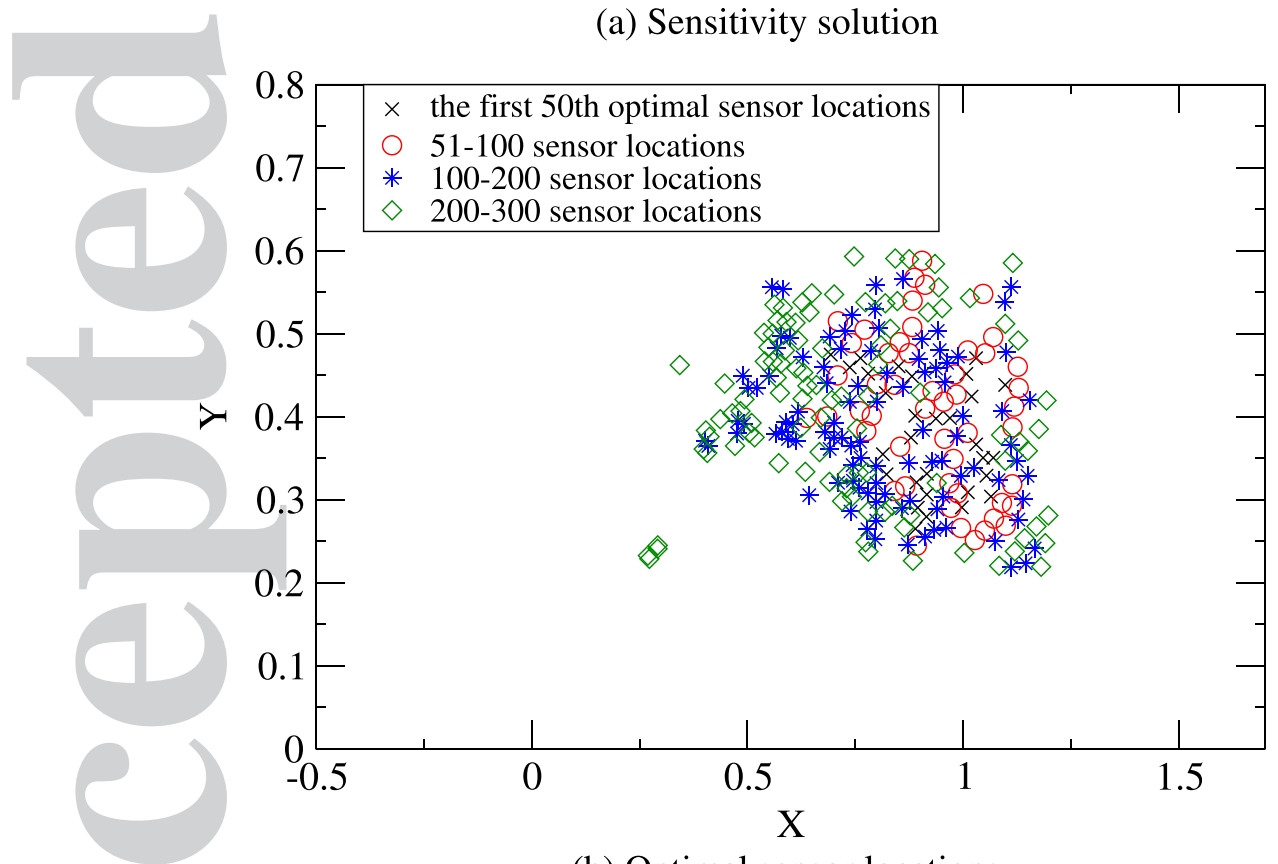

(b) Optimal sensor locations

Figure 6. Case 2: Optimisation of sensor locations. (a) adjoint sensitivity of the functional defined in (17) with respect to the solution at time instance $t=0.76$; (b) optimal sensor locations.

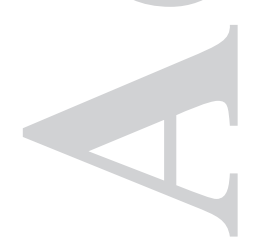

This article is protected by copyright. All rights reserved. 